\title{
Preliminary Investigations into the Utilization of Electron Tomography and Immunogold Immunocytochemistry in the Diagnosis of Mitochondrial Disease in Muscle
}

\author{
C.A. Ackerley*, J.M. Cameron**, B.A. Minassian***, C. Hawkins* and B.H. Robinson** \\ *Division of Pathology, Department of Paediatric Laboratory Medicine, Hospital for Sick Children, \\ Toronto, Canada M5G1X8 \\ **Research Institute, Hospital for Sick Children, Toronto, Canada M5G1X8 \\ ***Division of Neurology, Department of Paediatrics, Hospital for Sick Children, Toronto, Canada \\ M5G1X8
}

Mitochondrial myopathies comprise a clinically and etiologically diverse group of disorders caused by defects in cellular energy metabolism, including the breakdown of carbohydrates and fatty acids to generate adenosine triphosphate, predominantly through mitochondrial oxidative phosphorylation due to respiratory chain impairment. The wide clinical spectrum of mitochondrial myopathies ranges from severe infantile-onset multisystemic diseases to adult-onset isolated myopathies [1].

Typically in our laboratory, a muscle biopsy is divided into five pieces. One is frozen in isopentane and cryostat sections cut for histochemistry, another is frozen in liquid nitrogen for biochemical and mutational analysis studies, another is fixed in formalin and the remaining two fixed in either universal fixative for routine electron microscopy or $4 \%$ paraformaldehyde containing $0.1 \%$ glutaraldehyde for immunogold immunocytochemical studies. In biopsies from patients suspected of having a mitochondrial disorder, the cryostat sections are stained with modified Gomori's Trichrome, SDH, NADH and COX, biochemical assays are done for mitochondrial respiratory chain enzymes and sections prepared and examined using routine electron microscopy.

Mitochondria are distributed throughout the muscle cell primarily in the subsarcolemmal sarcoplasm and between the sarcomeres. The ultrastructure of mitochondria can vary in different regions of the same cell, in different cells of the same fibre type and from fibre type to fibre type even in normal muscle. This can be explained in part by the plane of the section and the orientation of the mitochondria within the section. Therefore, in order to definitively identify a mitochondria myopathy using electron microscopy alone, mitochondria must be significantly altered. These changes can include "parking lot" inclusions within the mitochondria, electron dense spheroids within abnormal cristae, mitochondria with whorled cristae, the absence of cristae and mitochondria with highly condensed cristae [2].

When abnormal findings are seen in the histochemistry or biochemical results and no significant changes in mitochondrial structure are detected, electron tomography is done on mitochondria from several areas within the cells including the subsarcolemmal sarcoplasm, the mitochondria between the sarcomeres and both type I and II myofibres. Tomographic reconstructions of the tilt series from each of the mitochondria are then $\mathrm{Z}$ sectioned to detect any consistent subtle changes [Fig. 1].

When mitochondrial respiratory chain enzyme results are abnormal, but no pathology has been detected, immunogold labeling is done on ultrathin cryosections with antibodies against the defective enzyme [Fig.2 and 3] [3]. Gold particle density is then determined first qualitatively and if 
need be quantitatively to see if the immunocytochemical results confirm the biochemistry. Double labeling experiments and further electron tomography studies are planned to see if any structural relationships can be determined between these enzymes in both normal and diseased tissues.

\section{References}

[1] Bernard A. et al., Curr Neurol Neurosci Rep. (2010) 2118.

[2] Sarnat H.B. and Marin-Garcia J., Can J Neuro Sci (2005) 32152

[3] Tam E.W. Neuropediatrics (2008) 39(6) 328.

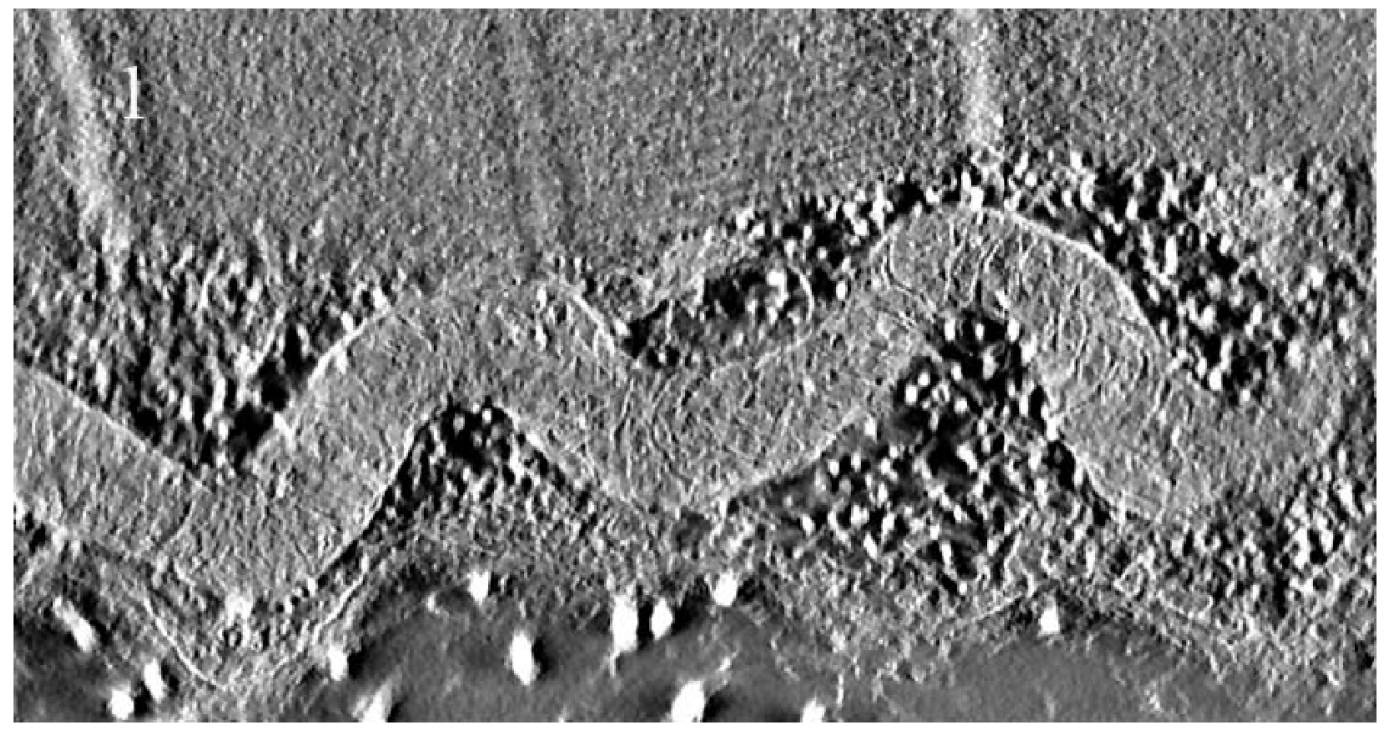

Fig.1 Z section of a mitochondria from a COX1 deficient patient. Note the serpentine shape and the mildly abnormal cristae. Picture width is $2 \mu \mathrm{m}$.
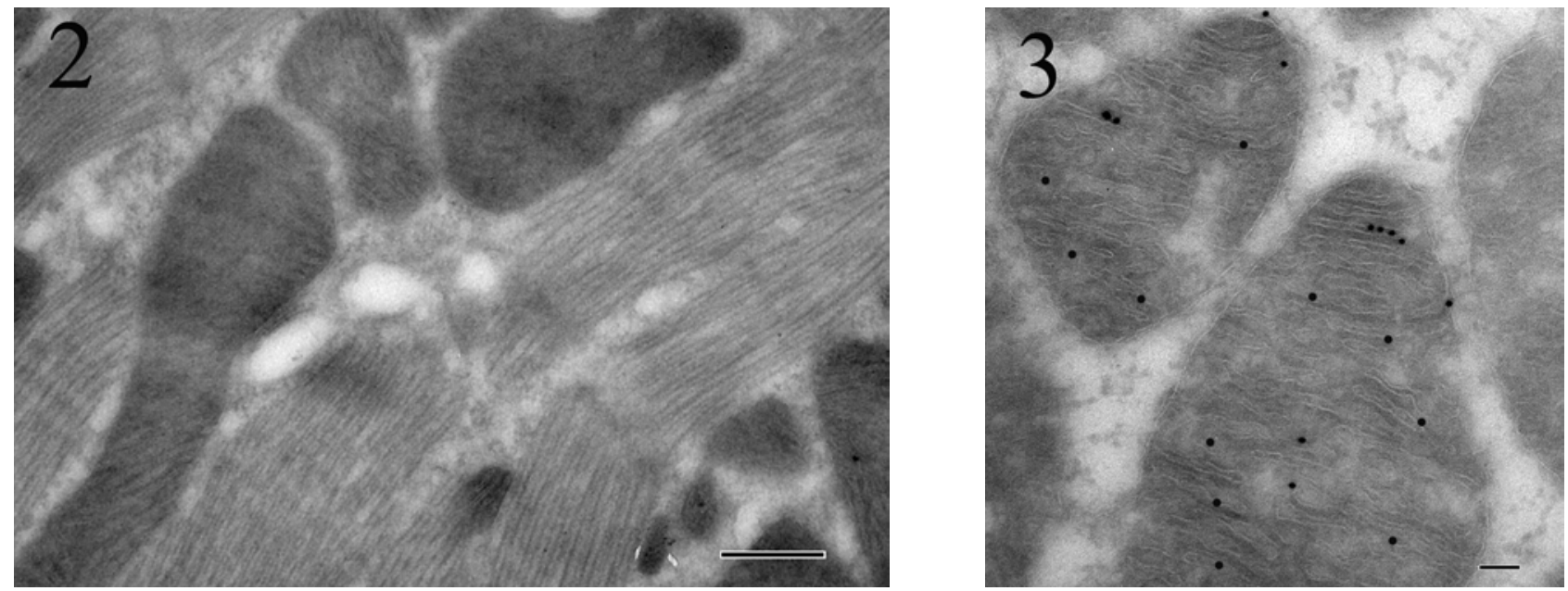

Fig.2 COX 1 immunogold labeled patient from Fig.1. Note the absence of any label. Bar equals 0.5 $\mu \mathrm{m}$.

Fig. 3 COX 1 immunogold labeled normal patient. Note the heavily decorated cristae. Bar equals 0.5 $\mu \mathrm{m}$. 